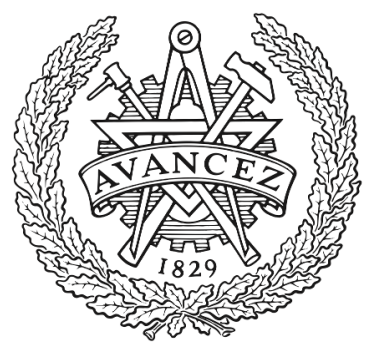

CHALMERS

UNIVERSITY OF TECHNOLOGY

\title{
Generation of single attosecond relativistic electron bunch from intense laser interaction with a nanosphere
}

Downloaded from: https://research.chalmers.se, 2023-04-26 14:09 UTC

Citation for the original published paper (version of record):

Horny, V., Veisz, L. (2021). Generation of single attosecond relativistic electron bunch from intense laser interaction with

a nanosphere. Plasma Physics and Controlled Fusion, 63(12).

http://dx.doi.org/10.1088/1361-6587/ac2996

N.B. When citing this work, cite the original published paper. 


\title{
Generation of single attosecond relativistic electron bunch from intense laser interaction with a nanosphere
}

\author{
Vojtěch Horný ${ }^{1,2, *}$ (i) and László Veisz ${ }^{3}$ (1) \\ ${ }^{1}$ Department of Physics, Chalmers University of Technology, Fysikgården 1, 41258 Göteborg, Sweden \\ ${ }^{2}$ LULI-CNRS; École Polytechnique, CEA; Université Paris-Saclay; UPMC Université Paris 06; \\ Sorbonne Université, Palaiseau cedex, F-91128, France \\ ${ }^{3}$ Department of Physics, Umeå University, 90187 Umeå, Sweden \\ E-mail: vojtech.horny@atlas.cz
}

Received 18 April 2021, revised 19 July 2021

Accepted for publication 23 September 2021

Published 12 November 2021

\begin{abstract}
Ultrahigh-intensity laser-plasma physics provides unique light and particle beams as well as novel physical phenomena. A recently available regime is based on the interaction between a relativistic intensity few-cycle laser pulse and a sub-wavelength-sized mass-limited plasma target. Here, we investigate the generation of electron bunches under these extreme conditions by means of particle-in-cell simulations. In a first step, up to all electrons are expelled from the nanodroplet and gain relativistic energy from time-dependent local field enhancement at the surface. After this ejection, the electrons are further accelerated as they copropagate with the laser pulse. As a result, a few, or under specific conditions isolated, pC-class relativistic attosecond electron bunches are generated with laser pulse parameters feasible at state-of-the-art laser facilities. This is particularly interesting for some applications, such as generation of attosecond $\mathrm{x}$-ray pulses via Thomson backscattering.
\end{abstract}

Keywords: optical injection, laser wakefield acceleration, electron bunch, perpendicular injection, crossing pulses

(Some figures may appear in colour only in the online journal)

\section{Introduction}

The interaction of intense laser pulses with matter is capable of accelerating electrons to ultrarelativistic energies via various techniques. Their oncoming applications comprise medical [1], industrial [2] or other disciplines of fundamental research [3]. It often relies on the conversion to secondary $\mathrm{x}$-ray radiation using phenomena such as bremsstrahlung emitted during propagation through a secondary solid target, betatron

\footnotetext{
Author to whom any correspondence should be addressed.
}

radiation produced during acceleration in a laser-plasma accelerator, undulator radiation by wiggling in an external magnetic field, or Thomson/Compton scattering on a second laser pulse $[4,5]$.

The acceleration techniques based on laser interaction with an underdense ${ }^{4}$ target are laser-wakefield acceleration [6] and direct laser acceleration [7]. The former is based on the generation of plasma waves in about a thousand to hundred times underdense plasma which sustains accelerating fields in the order of hundreds of $\mathrm{GV} \mathrm{m}^{-1}$ and enables electron energy gain up to GeVs [8] at a distance of centimeters, depending on the driving laser pulse and plasma parameters. The

\footnotetext{
4 The plasma is underdense when electron density $n_{\mathrm{e}}$ is lower than the critical density $n_{\mathrm{cr}}=\epsilon_{0} m_{\mathrm{e}} \omega^{2} / q_{\mathrm{e}}^{2}$ where $\varepsilon_{0}$ denotes vacuum permittivity, $m_{\mathrm{e}}$ and $q_{\mathrm{e}}$ electron mass and charge, respectively, and $\omega$ is the laser frequency.
} 
appropriate laser pulse is shorter than the plasma period ${ }^{5}$ and the typical measured electron bunch charge is up to hundreds of $\mathrm{pC}$ with a duration of a few femtosecond $[9,10]$. It is predicted that bunches with duration slightly shorter than a femtosecond can be generated when advanced injection techniques are employed [11-19].

Direct laser acceleration is suitable for longer laser pulses interacting with slightly underdense plasma which results in nC-class electron bunches [20-22] with a duration comparable with the accelerating laser pulse duration [23]. Electron bunches carrying a charge up to $\mu \mathrm{C}$ level, but of the duration in the order of picoseconds or longer can be generated via the self-modulated laser wakefield acceleration regime employing high energy laser systems, as it was recently shown at OMEGA [24].

High charge electron bunches can also be extracted from an overdense target and further accelerated in the form of bunch trains. Various methods based on laser interaction with a foil target under a large angle [25], nanofibre target [26], or generally with a target with sharp boundaries [27] have been predicted.

Some other proposed approaches do not rely on laser interaction with plasma. The longitudinal acceleration field of the laser pulse itself is in the order of $\mathrm{TV} \mathrm{m}^{-1}$ and can be used, under certain circumstances, for the acceleration of electron bunches. For example, a relativistic electron bunch already pre-accelerated in a conventional accelerator is further accelerated to significantly higher energies when co-propagating with the an intense laser pulse [28-30].

Additionally, during a linearly polarised laser pulse interaction with an overdense nano-droplet, one or two trains of attosecond electron bunches are emitted from its surface in the polarization plane every half [31] or full laser cycle [32], depending on whether the nano-droplet is initially placed at or out of the laser propagation axis, respectively. For circular polarization, an electron spiral structure is continuously swept from the droplet [33]. In a first step of the interaction with linear polarization, electron bunches are emitted with relativistic energy by the enhanced laser field from the surface of the nano-target. In a second step, they co-propagate with the laser pulse within the same optical cycle and gain further energy via vacuum laser acceleration.

In our configuration, the transverse component of the laser electric field accelerates the electrons and its magnetic field turns them forward. In this paper, we discuss the consequences on the resulting electron bunch properties such as its continuous energy spectrum and the maximum electron energy. Furthermore, we suggest a scheme providing a single attosecond electron bunch with currently available technology, employing few-cycle pulses and mass-limited nano-droplet targets. We also describe the Thomson backscattering resulting in attosecond $\mathrm{x}$-ray radiation as a possible application.

\footnotetext{
${ }^{5}$ The plasma period is $T=2 \pi / \omega_{\mathrm{p}}$, where $\omega_{\mathrm{p}}$ is the plasma frequency defined as $\omega_{\mathrm{p}}=\sqrt{\frac{n_{\mathrm{e}} q_{\mathrm{e}}^{2}}{\epsilon_{0} m_{\mathrm{e}}}}$
}

\section{Simulation results and their analysis}

We illustrate the concept using 3D particle-in-cell (PIC) simulations. These simulations capture the irradiation of the droplet by the laser pulse, consequent proton and electron dynamics, and the resulting electron acceleration processes. We use the SMILEI PIC code [34] which enables accurate representation of the tightly focused laser pulse by Maxwell-consistent description of fields [35] at the left boundary. The laser pulse is Gaussian in both temporal and spatial domain. In the focal plane $x_{\mathrm{f}}=1 \mu \mathrm{m}$, its electric field is given as

$$
\begin{aligned}
\mathbf{E}= & E_{0} \exp \left(-\frac{2 \ln 2 r^{2}}{D^{2}}\right) \exp \left[-\frac{2 \ln 2\left(t-t_{0}\right)^{2}}{\tau^{2}}\right] \\
& \times \cos \left[\omega\left(t-t_{0}\right)+\varphi_{\mathrm{CEP}}\right] \mathbf{e}_{y}
\end{aligned}
$$

where $E_{0}=a_{0} m_{\mathrm{e}} c \omega / q_{\mathrm{e}}$ is the electric field amplitude, $m_{\mathrm{e}}$ is the electron rest mass, $c$ is the speed of light in vacuum, $\omega=2 \pi c / \lambda$ is the laser frequency, $\lambda=0.75 \mu \mathrm{m}$ is the laser wavelength, $q_{\mathrm{e}}$ is the elementary charge, $a_{0}=$ $0.855 \sqrt{I\left[10^{18} \mathrm{~W} \mathrm{~cm}^{-2}\right]} \lambda[\mu \mathrm{m}]=6.4$ is the laser strength parameter connected to the peak laser intensity $I=10^{20} \mathrm{~W} \mathrm{~cm}^{-2}$, $\tau_{0}=3.8 \mathrm{fs}$ is the pulse duration in the sense of full width at half maximum (FWHM) of intensity, $r=\sqrt{y^{2}+z^{2}}, D=1.2 \mu \mathrm{m}$ is the spot size (FWHM of intensity), $\varphi_{\mathrm{CEP}}=0$ is the carrierenvelope phase in the focus and $\mathbf{e}_{y}$ is the unit vector in the $y$ direction.

The pre-ionized plasma target is located at $x=x_{\mathrm{f}}, y=$ $z=0$. The peak laser intensity hits the focal plane at the time $t_{0}=10.8 \mathrm{fs}$. The target is represented as a hydrogen plasma sphere with radius $R=50 \mathrm{~nm}$ and electron density $n_{\mathrm{e}}=100 n_{\mathrm{cr}}$, where $n_{\mathrm{cr}}=1.98 \times 10^{21} \mathrm{~cm}^{-3}$ is the classical critical density for our wavelength. The target is a sharp sphere as the influence of possible plasma expansion before the interaction was already discussed in [36].

The simulation box dimensions are $12 \mu \mathrm{m} \times 10 \mu \mathrm{m}$ $\times 10 \mu \mathrm{m}$ and the grid resolution is 100 cells per wavelength. Approximately $2.0 \times 10^{6}$ computational particles of every species are simulated.

The first stage of the process is the ejection of the electron bunches from the target. The laser pulse extracts the electron bunches from the sub-micron droplet twice every optical cycle as in [31]. The bunches remain mainly in the polarization plane of the pulse, the bunches of each cycle are ejected in mutually opposite directions. The reason for the ejection is the enhanced electrostatic field near the target surface. The enhanced field together with the electron density is depicted in figure 1. A movie of the process can be found in the supplementary materials (available online at stacks.iop.org/PPCF/ 63/125025/mmedia).

At time $t_{0}$, the peak of the laser field $E_{0}$ reaches the sphere, which results in the ejection of a new electron bunch and the maximum of field enhancement. In this case, the field strength is as high as $2.3 E_{0}=63 \mathrm{TV} \mathrm{m}^{-1}$. This enhancement factor, which has a theoretical maximum of 3 in a highly overdense plasma [31], is much smaller than in traditional nanophotonics with enhancements in the range of 100-1000, because in 


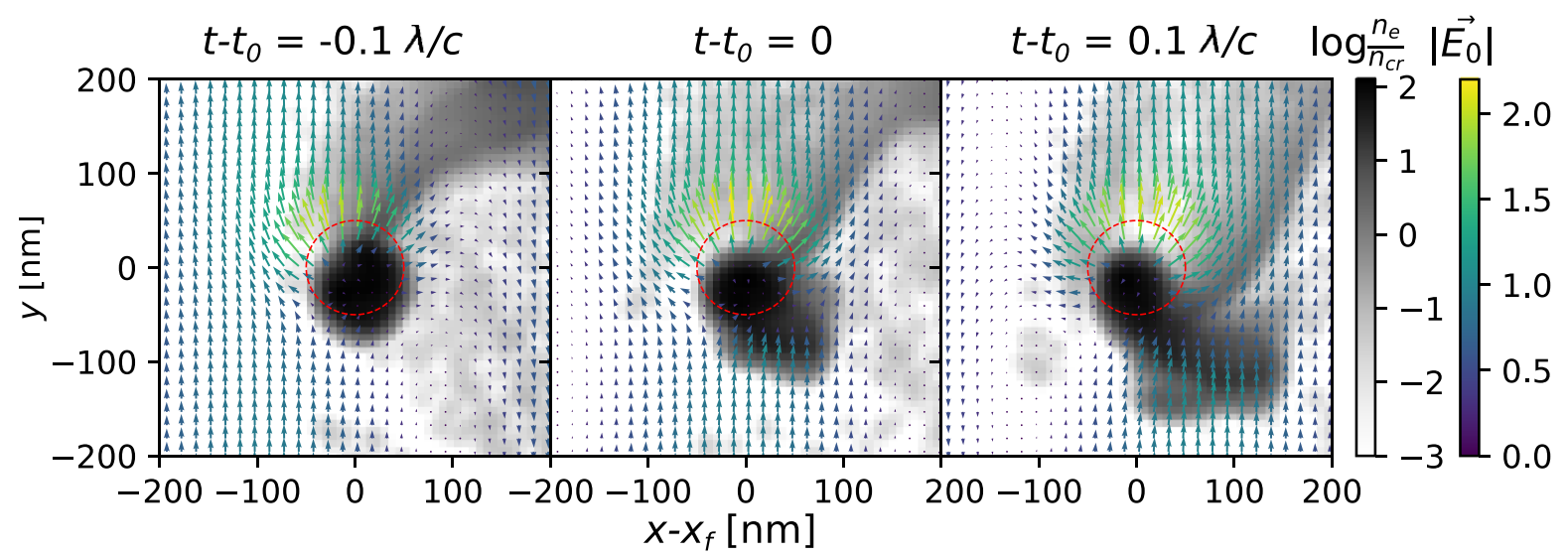

Figure 1. Process of electron ejection and field enhancement. Grey shades—logarithm of electron density, color arrows—electric field, red circle-initial location of the target. The electric field colorbar is normalized to the peak field in vacuum. Full movie is in supplementary materials. Parameters: $R=50 \mathrm{~nm}, n_{\mathrm{e}}=100 n_{\mathrm{cr}}, I=10^{20} \mathrm{~W} \mathrm{~cm}^{-2}, \lambda=0.75 \mu \mathrm{m}, D=1.2 \mu \mathrm{m}, \tau_{0}=3.8 \mathrm{fs}, \varphi_{\mathrm{CEP}}=0$, and $t_{0}$ is the time the laser peak hits the center of the target.

plasmas, the excitation of the localized surface plasmon is far from resonance. In general, the laser electric field is enhanced on both sides of the target. However, as a considerable number of electrons are removed from our mass-limited target, a significant screening effect is observed at the electron emission side [36]. In our case, the field enhancement on the electron emission side of the target reaches the value of $1.12 E_{0}$.

The Mie theory [37] predicts that for sufficiently high densities $\left(>n_{\text {cr }}\right)$, the maximum field enhancement factor is equal to 3 ; however, it also assumes very small targets $(2 \pi R \ll$ $\lambda)$ and non-relativistic intensities $\left(a_{0} \ll 1\right)$. This limit for the enhancement factor was confirmed in simulations even for $a_{0}>1$ and densities $>n_{\mathrm{cr}} a_{0}[31,38]$. Nevertheless, the enhanced field accelerates the electrons to the energies of several MeVs at distances lower than one micron [36]. These ejected electrons become relativistic and can co-propagate with the laser pulse, where the second stage of the acceleration takes place.

\subsection{Second stage acceleration mechanism}

We will show that the dominant acceleration mechanism within this stage is transverse acceleration by the transverse laser electric field and a consequent bending in a forward direction by its magnetic field rather than the longitudinal component of the tightly focused laser pulse. This effect was neither discussed, nor explained in previous works $[31,36,38]$, however, it has relevant consequences. Figure 2 shows the trajectories of two macroparticles of the electron bunch ejected at $t=t_{0}$. The left column represents a typical electron which reaches a lower final energy of $3.5 \mathrm{MeV}$, the right column depicts a typical electron from the high energy tail of the spectrum. The upper panels show the evolution of the $\mathbf{E} \cdot \mathbf{v}$ scalar products for both selected electron macroparticles; these are the fields and velocities that are used for the integration of their equation of motion within the particle-in-cell loop.

As shown in [7, 39], the relativistic electrons gain energy from the laser electric field. Integrating the energy equation $d\left(\gamma m c^{2}\right) / d t=q_{\mathrm{e}} \mathbf{E} \cdot \mathbf{v}$ along the electron trajectory provides the change of the electron energy

$$
W=q_{\mathrm{e}} \int \mathbf{E} \cdot \mathbf{v} \mathrm{d} t
$$

clearly indicating that magnetic force cannot influence this energy. Comparing the first two rows in figure 2, a clear correlation between the temporal integral of $-\mathbf{E} \cdot \mathbf{v}$ and the temporal profile of the kinetic energy of electrons $E_{k, e}$ is apparent. In particular, as we can see, the electron is dominantly accelerated in the time interval when $\left|E_{y} v_{y}\right|>\left|E_{x} v_{x}\right|$, suggesting the dominant role of the transverse electric field component for the acceleration. The rate of the electron energy gains and losses is proportional to the sum $-\left(E_{x} v_{x}+E_{y} v_{y}\right)$, neglecting the insignificant contribution of the third coordinate. For example, particle energy in the right column growths from 0 to $12.5 \mathrm{MeV}$ during $15 \mathrm{fs}$, which corresponds to the average accelerating rate of $0.83 \mathrm{MeV} \mathrm{fs}^{-1}$. This is exactly the average of $q_{e}\left(E_{x} v_{x}+E_{y} v_{y}\right)$ for that time interval. The average accelerating field during this time corresponds to about $3 \mathrm{TV} \mathrm{m}^{-1}$. The third and fourth rows show the electron positions and velocities, respectively.

Figure 3 visualises the acceleration mechanism approximately at the distance of one Rayleigh length from the target, which is $4.4 \mu \mathrm{m}$ in our case. Panels a and c depict the spatial distribution of the electron density together with the longitudinal electric field $E_{x}$ and azimuthal magnetic field $B_{z}$, respectively. The electron bunches are mainly located in the regions with the positive value of $E_{x}$, where they are decelerated by the longitudinal field. This apparent discrepancy is explained by the mechanism of the electron ejection from the target.

Panel b shows the map of electron energy at the background of the transverse electric field representing the laser pulse. Comparing panels $\mathrm{b}$ and $\mathrm{c}$, the crucial role of the magnetic component of the Lorentz force on the turning the electrons in the forward direction is confirmed. For an illustration, the most energetic electrons within the half-space $y<0$ where $v_{y}<0$ are located at the regions with the positive $B_{z}$, in particular, 


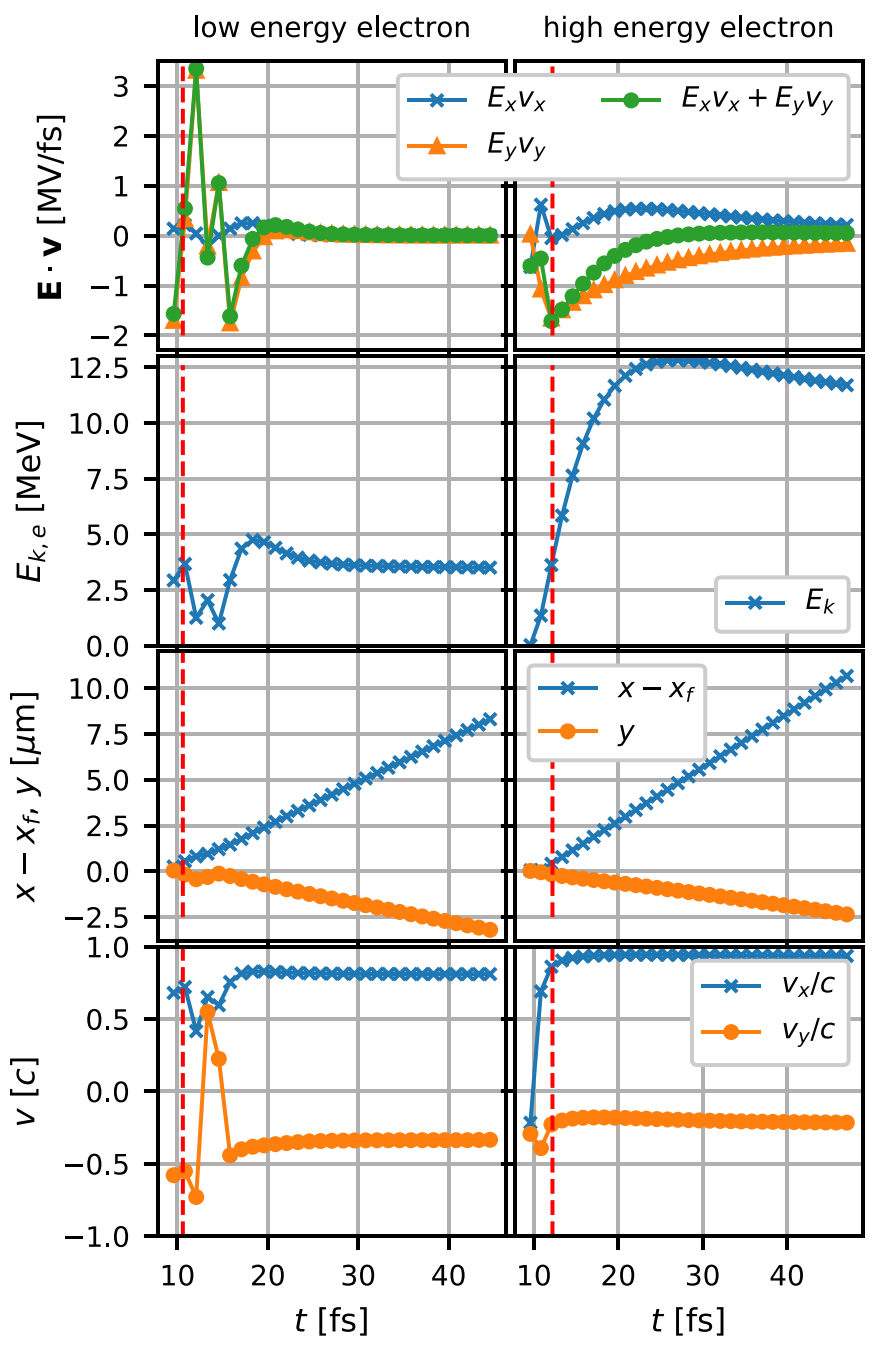

Figure 2. Second acceleration stage trajectories of two characteristic macroparticles from the most energetic bunch and the correlation of the particle energy evolution with the $\mathbf{E} \cdot \mathbf{v}$ product. First row: time profiles of $E_{x} v_{x}$ (blue, (x), $E_{y} v_{y}$ (orange, triangle) and their sum (green, circle). Second row: evolution of the kinetic energy of electron. Third row: electron position. Fourth row: electron velocity. Vertical red dashed lines mark the time when electron is $0.5 \mu \mathrm{m}$ from the initial target location, signalizing the approximate time, when the electrons enter the second stage. It shows that the initial electron energy in the second stage is relativistic.

at its rear edges. Due to the negative charge of the electron, the magnetic force component $q_{\mathrm{e}} v_{y} B_{z}$ pushes them forward. A movie of this acceleration and bending process extended by the visualisation of electron energy together with the fields can be found in the supplementary materials (available online at stacks.iop.org/PPCF/63/125025/mmedia).

\subsection{Continuous energy spectrum}

The fact that longitudinal velocity of electrons is lower than the phase velocity of the laser pulse together with the bunch orientation in space causes the different parts of a single bunch to experience significantly different acceleration fields; some parts of the bunch are even decelerated. As a result, the initial spectrum (shown as orange curve in figure 4(a)) evolves into a spectrum with a rather long high energy tail with a characteristic cut-off energy. As can be seen in figure 3(a) or (b), the longitudinal extent of each single electron bunch during and after acceleration is still lower than $100 \mathrm{~nm}$, i.e., $<300$ as.

To determine the scaling with laser strength parameter and the maximum electron energy gain, the second stage of acceleration is treated as an interaction of a free electron in a laser field with a certain initial energy defined by the first step. The initial momentum is assumed to be perpendicular to the laser propagation direction as typical for Mie scattering from nanoparticles with a size much smaller than the laser wavelength and the initial energy is relativitic $\left(\gamma_{0} \gg 1\right)$. Under these conditions, the maximum electron energy is calculated to be

$$
\max \gamma=\gamma_{0}+a_{0}+a_{0}^{2} / 2 \gamma_{0}
$$

There is an important conclusion about the scaling with the laser field. As $\gamma_{0}$ is a function of $a_{0}$, the exact scaling might be complex. However, taking into account our typical initial (field dependent) electron energies from the first step $\left(\gamma_{0} \sim 5-11\right)$ and the laser field strengths $\left(a_{0} \sim 3-20\right)$, the last term is small compared to the others and the dominant term is the second, field dependent one. If $\gamma_{0} \propto a_{0}$, then $\max \gamma \propto a_{0}$. These also explain the former simulation results for scaling in the supplementary information of [36]. It should be noted that the electrons can indeed gain energy from the laser as they are injected temporally in the middle of the laser pulse around the peak intensity and thus the assumptions of Lawson-Woodward theorem are not fulfilled at all. The angular distribution is similar to the the scattering angle of an originally resting electron in the laser field in vacuum [38], but is modified a bit by the initial electron transverse momentum.

The evolution of the electron energy spectra is shown in figure 4 for our basic parameters and for the case with larger target and more intense and longer laser pulse. The low energy peaks below $1 \mathrm{MeV}$ correspond to electrons which remained in target. The high energy tail reaches up to $15.5 \mathrm{MeV}$ and $49.5 \mathrm{MeV}$, respectively. The energy from the first step is several times lower $(<5 \mathrm{MeV})$ than from the second step and scales approximately with $a_{0}$ in contrary to $\sqrt{a_{0}}$ in [36]. The actual maximum energy gain at the end is limited by the dephasing. The final energies are in reasonable agreement with the maximum electron energy scaling with $a_{0}$ according to equation (3). The dynamics of the energy spectra evolution in both cases shows initial intensive acceleration with $>5 \mathrm{TV}$ $\mathrm{m}^{-1}$ field which steadily slows down and vanishes.

Detailed analysis showed that electrons of the high energy tail of the distribution leave the accelerating phase, i.e. dephase and get decelerated. Afterwards, the maximum energy remains similar as the contribution of the most energetic electrons to the total spectrum is steadily being replaced by their in a moment slightly lower energy predecessors located in front of them. Those electrons are, actually, still in the acceleration phase. Such acceleration and deceleration process lasts until the whole bunch is transversely scattered out of the high intensity region which occurs at the distance similar to Rayleigh length, as it can be seen in figure 3(b) or alternatively the whole bunch is dephased. 

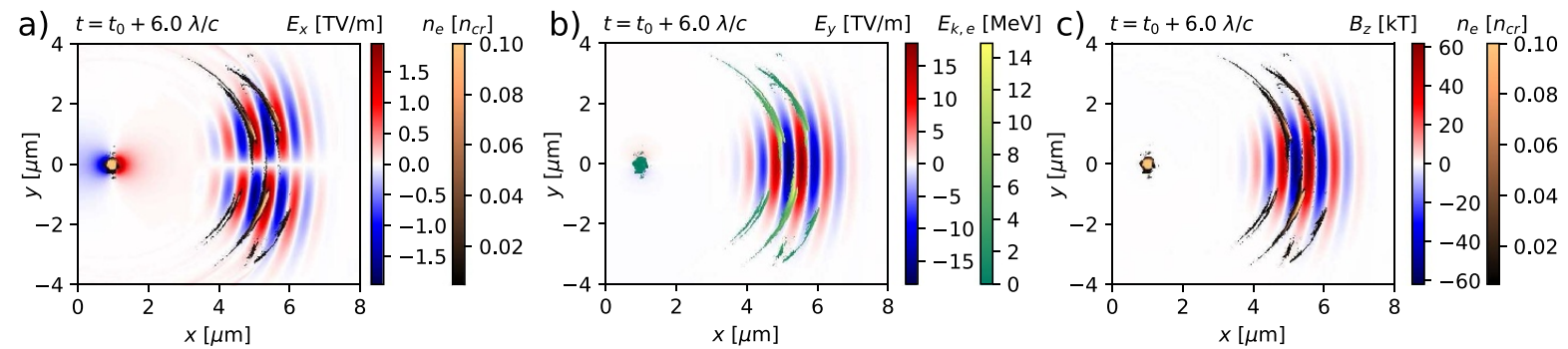

Figure 3. Positions of the laser pulse and electron bunches six optical cycles after the pulse peak hit the target. (a) Electron density $n_{\mathrm{e}}$ and longitudinal electric field $E_{x}$. (b) Average electron energy $E_{k, e}$ and transverse electric field $E_{y}$. (c) Electron density $n_{e}$ and azimuthal magnetic field $B_{z}$. In all panels, only cells with $n_{\mathrm{e}}>0.005 n_{\text {cr }}$ are shown for $n_{\mathrm{e}}$ and $E_{k, e}$ plots. Simulation parameters: $R=50 \mathrm{~nm}$, $n_{\mathrm{e}}=100 n_{\mathrm{cr}}, I=10^{20} \mathrm{~W} \mathrm{~cm}^{-2}, D=1.2 \mu \mathrm{m}, \tau_{0}=3.8 \mathrm{fs}$.
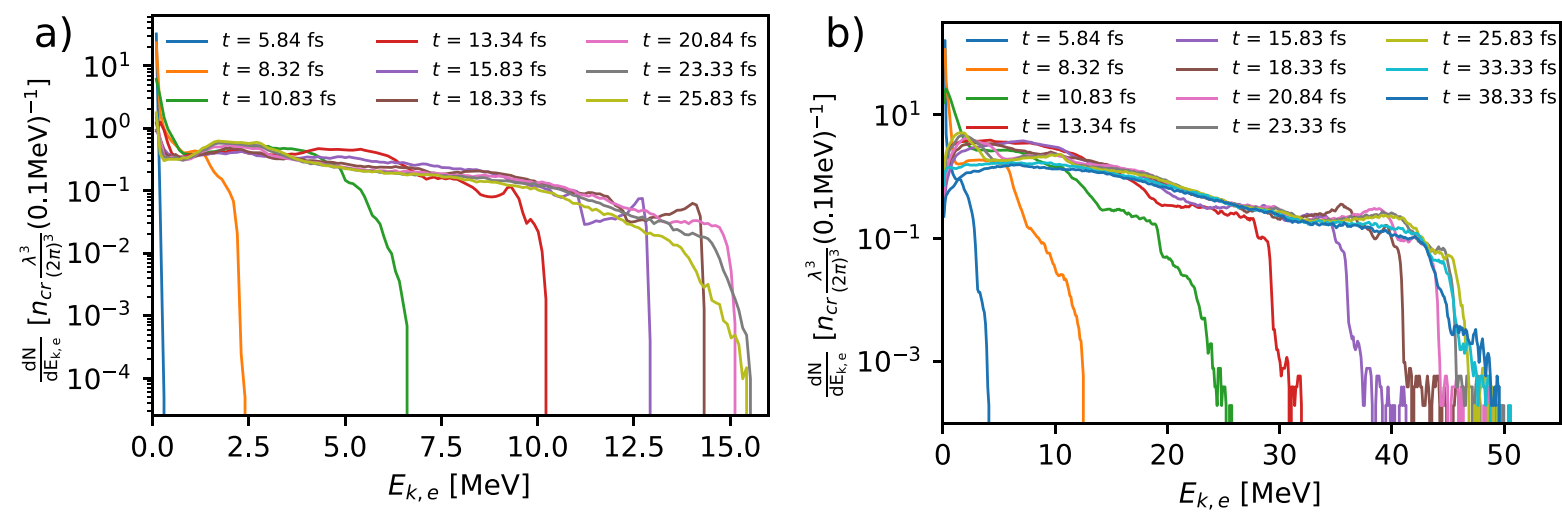

Figure 4. Energy spectra evolution for parameters of figure 3 (a) and for $R=100 \mathrm{~nm}, n_{\mathrm{e}}=100 n_{\mathrm{cr}}, I=10^{21} \mathrm{~W} \mathrm{~cm}{ }^{-2}, D=1.2 \mu \mathrm{m}$, $\tau_{0}=5.0 \mathrm{fs}(\mathrm{b})$.

\subsection{Spectra of respective attosecond bunches and generation of a single bunch}

The spectral shape connected with the respective single bunches are similar to each other and to the total spectrum, as shown in figure 5 . The high energy cutoff is given by the local intensity of the laser pulse at the specific position of the bunch, corresponding to the scaling set by equation (3). The electron bunches are separated by one laser period ( $2.5 \mathrm{fs})$ and have duration of about 200 as (in figure 3, the duration of the main bunch considered as the FWHM of the density is 210 as). Our simulations show that typically, the conversion efficiency of laser pulse energy into the kinetic energy of all electrons is around $1 \%$.

Let us derive the relative cut-off energy $E_{c, m}$ of $m$-th bunch for $\varphi_{\mathrm{CEP}}=0$. The pulse temporal envelope is

$$
a(t)=a_{0} \exp \left[-\frac{2 \ln 2\left(t-t_{0}\right)^{2}}{\tau_{0}^{2}}\right] .
$$

Electron bunches are emitted from the target every laser half period at the time moments $t_{\mathrm{m}}=t_{0}-m \lambda / 2 c$ where $m \in$ $[0, \pm 1, \pm 2, \pm 3, \ldots]$. Because $E_{c, m}$ is proportional to the corresponding value of $a$, it can be written

$$
\frac{E_{c, m}}{E_{c, 0}}=\exp \left[-\frac{\ln 2(m \lambda)^{2}}{2 c^{2} \tau_{0}^{2}}\right],
$$

where $E_{c, 0}$ is the energy of the bunch ejected at the peak of the laser pulse. This cut-off energy ratio strongly depends on the pulse duration, shortening of the laser pulse duration leads to a higher difference in energies of subsequent bunches.

In our case, equation (5) predicts the cut-off energies of bunches \pm 2 from figure 5 to be by a factor of 0.55 lower than the cut-off energy of bunch No. 0. This and other predictions about ratios of energy cut-off values for respective bunches are represented by vertical lines in figure $5(\mathrm{~d})$, setting the energy of bunch No. 0 to $15.6 \mathrm{MeV}$. This agreement and also the data for higher intensity shown in figure 4 suggest the scaling for the cut-off energy with laser strength parameter as

$$
E_{c, m}(\mathrm{MeV}) \approx 2.4 a_{0}
$$

This is in reasonable agreement with equation (3), especially for $\gamma_{0} \propto a_{0}$. The aforementioned electron bunch No. 0 from figures 5(a) and (d) is relatively well isolated with significantly higher energy than its neighbouring bunches \pm 2 , considering a limited solid angle, here for example the lower half plane. Furthermore, the isolation of this most energetic electron bunch with the fixed laser pulse duration can be increased by proper tuning of the total electron number in the target which is set by its size and density. In the optimal configuration all the electrons are evacuated from such a mass-limited target during the first half of the laser pulse. As a result, a well isolated electron bunch is ejected in the lower half-space (figure 5(b)) or even better isolated single electron bunch with 
a)

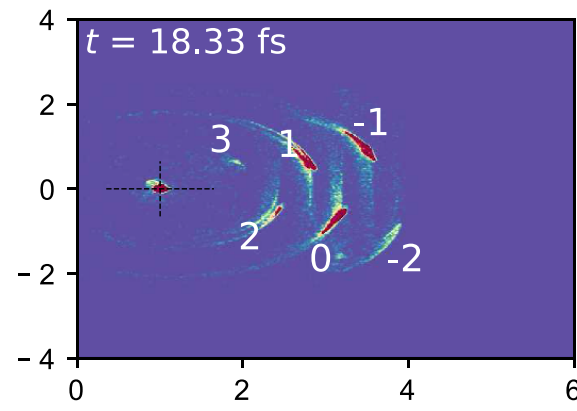

$n_{e}=100 n_{c r}$

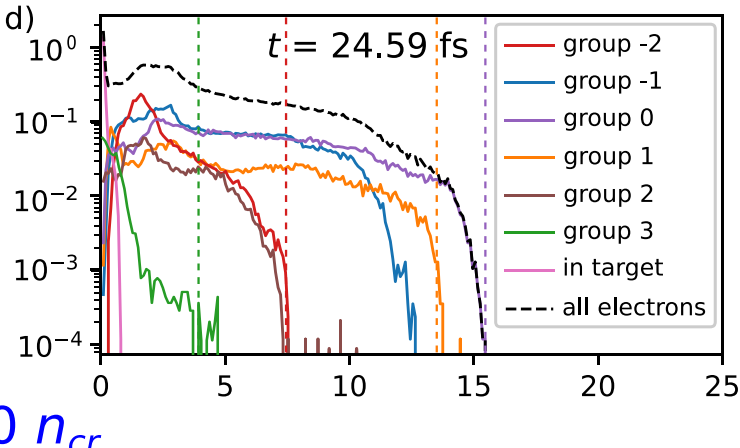

$n_{e}=70 n_{c r}$ b)

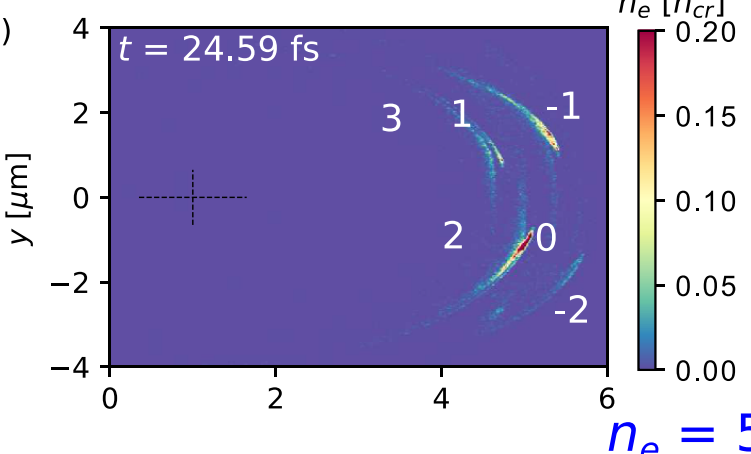

c)

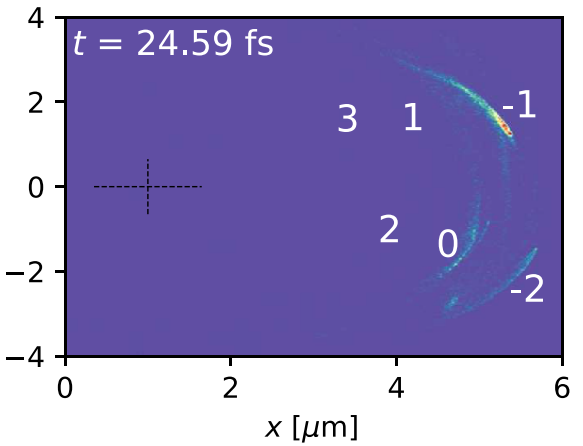

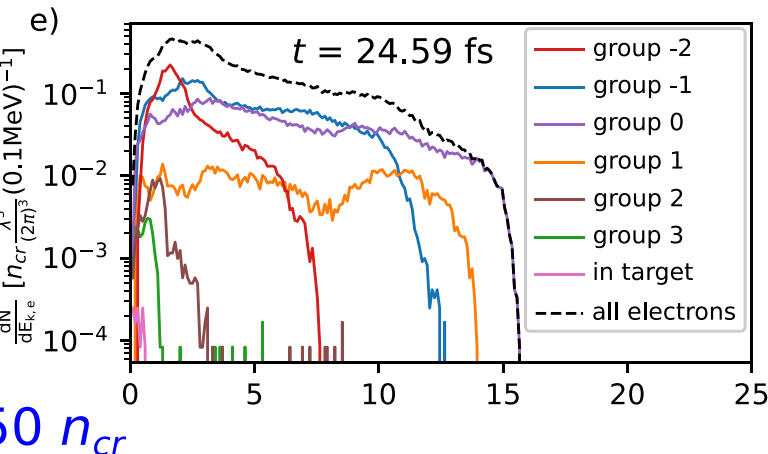

f)

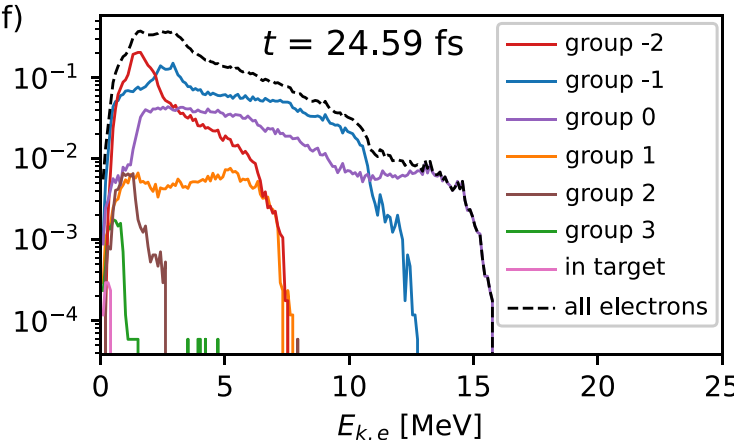

Figure 5. Electron density distribution ((a)-(c)) and energy spectra of single electron bunches ((d)-(f)) for different target densities. Common parameters: $R=50 \mathrm{~nm}, I=10^{20} \mathrm{~W} \mathrm{~cm}^{-2}, D=1.2 \mu \mathrm{m}, \tau_{0}=3.8 \mathrm{fs}$. Color dashed lines in panel (d) illustrated the expected cut-off energy ratios according to equation (5).

reduced energy is emitted in the upper half-space, as is shown in figure 5(c). This latter single bunch corresponds to the blue line in the related spectrum in figure 5(f). As this bunch travels with the leading edge of the laser pulse where its intensity does not reach its maximum yet, it does not comprise the most energetic electrons. Nevertheless, a single isolated relativistic attosecond electron bunch with a continuous spectrum reaching up to $12.5 \mathrm{MeV}$ is generated with the laser pulse parameters similar to [36].

Table 1 shows the charges of respective bunches for several set of parameters. It also suggest the conditions for the single bunch generation which are:

- The trivial case is when the laser pulse is short enough that it captures only one considerably populated bunch in the given half-space. Practically, it is fulfilled when $\tau_{0} \lesssim 1.3 \lambda / c$, see the results for $\tau_{0}=3$ fs. In the same manner, single electron attobunch can be generated employing different configurations of the laser pulse interaction with the overdense target [40, 41].

- In the case of longer pulses, another option is controlling the laser intensity. In the lower half-space, the main bunch (No. 0 ) has just slightly less electrons, while the preceding and following bunches (No. \pm 2 ) have much less at a reduced $I=2.5 \times 10^{19} \mathrm{~W} \mathrm{~cm}^{-2}$ intensity and $70 n_{\mathrm{cr}}$ density compared to the higher intensity. This naturally provides a way to get better isolated electron bunches by reducing the laser intensity, though other parameters, e.g. electron energy, are also influenced.

- A further option is to use a limited total electron number in the target with parameters of the target and laser pulses complementing each other. Using the original short laser pulse, the total charge of the target electrons must be smaller than the sum of charges carried by the first upper bunch and first 
Table 1. Charge in respective bunches in dependence on target density and laser intensity. $I_{18}$ is the intensity in units of $10^{18} \mathrm{~W}^{\mathrm{cm}}{ }^{-2}$, $D=1.2 \mu \mathrm{m}, R=50 \mathrm{~nm}, Q_{\mathrm{t}}$ is charge left in the target.

\begin{tabular}{|c|c|c|c|c|c|c|c|c|c|}
\hline$n_{0}\left(n_{\mathrm{cr}}\right)$ & $I_{18}$ & $\tau_{0}(\mathrm{fs})$ & $Q_{-1}(\mathrm{pC})$ & $Q_{1}(\mathrm{pC})$ & $Q_{3}(\mathrm{pC})$ & $Q_{-2}(\mathrm{pC})$ & $Q_{0}(\mathrm{pC})$ & $Q_{2}(\mathrm{pC})$ & $Q_{t}(\mathrm{pC})$ \\
\hline 50 & 25 & 3.8 & 1.356 & 1.264 & 0.123 & 0.261 & 1.815 & 0.487 & 1.122 \\
\hline 70 & 25 & 3.8 & 1.462 & 1.351 & 0.159 & 0.278 & 1.957 & 0.509 & 3.778 \\
\hline 50 & 100 & 3.8 & 3.342 & 0.181 & 0.011 & 1.866 & 1.498 & 0.037 & 0.001 \\
\hline 70 & 100 & 3.8 & 3.960 & 0.560 & 0.018 & 1.959 & 3.314 & 0.051 & 0.002 \\
\hline 100 & 100 & 3.8 & 4.304 & 1.801 & 0.249 & 2.071 & 4.046 & 0.909 & 0.973 \\
\hline 100 & 100 & 3.0 & 3.850 & 2.039 & 0.067 & 0.649 & 4.519 & 0.524 & 1.676 \\
\hline
\end{tabular}

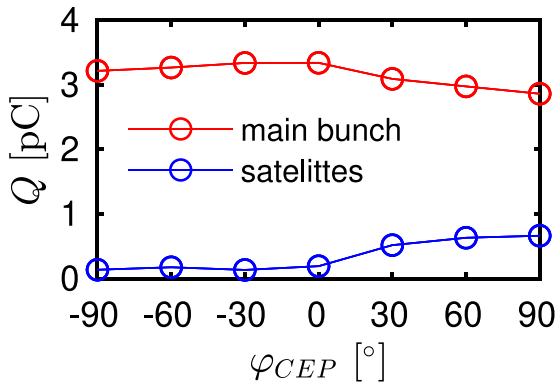

Figure 6. Charge in the main bunch and total charge in all satellite bunches in the upper half-space in dependence on the carrier-envelope phase. $\varphi_{\mathrm{CEP}}=0$ means the cosine pulse. $R=50 \mathrm{~nm}, n_{\mathrm{e}}=50 n_{\mathrm{cr}}, I=10^{20} \mathrm{~W} \mathrm{~cm}^{-2}, D=1.2 \mu \mathrm{m}, \tau_{0}=3.8 \mathrm{fs}$

two bottom bunches. Under such circumstances, the second upper bunch is not ejected as there are no electrons left in the target.

In the latter case, it is reasonable to tune the ratio between the charges of the respective bunches by adjusting the CEP, while in the former cases $\varphi_{\mathrm{CEP}}=0$ is the optimum. Figure 6 shows this dependence of the charge in the dominant bunch in the upper half-space and the sum of its satellite bunches on CEP for mass-limited target from figure 5(c). The best ratio between those is achieved for $\varphi_{\mathrm{CEP}}=-30^{\circ}$, more than $96 \%$ of the charge accelerated in the upper half-space is comprised by the main bunch. In such a case, the isolation of the single bunch is not significantly sensitive to the accurate CEP finetuning of the laser pulse.

The CEP-tuning also sets the highest energy of the dominant bunch. Figure 7 compares the simulations with $\varphi_{\mathrm{CEP}}=$ $-30^{\circ}$ and $90^{\circ}$. The second simulation sets the highest energy of $14.9 \mathrm{MeV}$ for the main bunch which, however, comprises only $81 \%$ of the charge accelerated in the upper half-space now. The shape of the total spectrum and maximal electron energy are similar in both cases.

\section{Discussion}

\subsection{Attosecond bunch with continuous spectrum}

The presented acceleration scheme represents a promising path for generation of isolated attosecond electron bunches. Its duration at generation is principally restricted to $\tau<\lambda / 2 c$, which is $1.25 \mathrm{fs}$ in our case, however, practically, it is only a fraction of it ( $\sim 200$ as). The price of this asset is giving up the generation of monoenergetic bunches. In the first phase of interaction, electron bunches are ejected from the target with the periodicity of $\lambda / 2 c$ and moderately relativistic energy $(\sim \mathrm{MeV})$. It means that the initial length of bunches is already comparable to the length of the steep acceleration structure. Inevitably, the respective parts of the bunch are accelerated by significantly different acceleration fields which results in a continuous spectrum.

In fact, the broad energy spread is a high price as it significantly elongates the attosecond bunches after a short $(\sim 100 \mu \mathrm{m})$ propagation as simulations indicate. Furthermore, space-charge effects (Coulomb explosion) from below $1 \mathrm{pC}$ bunch charge ( $\sim 10^{7}$ electrons) can further increase this elongation. However, a big advantage of the mass-limited target is the control over the number of electrons and thus the Coulomb explosion of the bunch after the interaction. Additionally, depending on the $f$-number of laser focusing, the laser can mitigate this elongation on a few Rayleigh range spatial scale, but afterwards it dominates. Therefore, the attosecond bunches, without extra measures, should be utilized within $\sim 100 \mu \mathrm{m}$ from the generation point.

\subsection{Feasibility}

The suggested scheme of single electron bunch generation is feasible with several contemporary laser systems. The first experiment in this direction has even been realized providing bunch charges up to $100 \mathrm{pC}$ [36], which was based on a special system reported by Rivas et al [42]. It is a synthesizer which uniquely combines ultra-relativistic focused intensities of about $10^{20} \mathrm{~W} \mathrm{~cm} \mathrm{~cm}^{-2}$ with a pulse duration of sub-two carrier-wave cycles and ultrahigh temporal contrast. However, several other lasers are good candidates for the demonstration of the scheme or at least for the preliminary experiments. They are providing among others a $10 \mathrm{~Hz}$ repetition rate, $75 \mathrm{~mJ}$ energy and 4.7 fs duration laser pulses [43], $42 \mathrm{~mJ}$ with less than $7 \mathrm{fs}$ and a peak intensity of $6.9 \times 10^{19} \mathrm{~W} \mathrm{~cm}^{-2}$ [44], or at $1 \mathrm{kHz}$ repetition rate $5 \mathrm{~mJ}, 3.4$ fs laser pulses with an estimated vacuum laser intensity of $\approx 5 \times 10^{18} \mathrm{~W} \mathrm{~cm}^{-2}$ [45], $32 \mathrm{~mJ}$ with less than 7 fs [46].

As the target, commercially available nanoparticles or nanospheres with various selectable diameters are very suitable [47]. Although, their precise 3D positioning is still a challenging task, a promising solution is offered by Paul traps, as experimentally demonstrated for this purpose in [48, 49]. 
a)

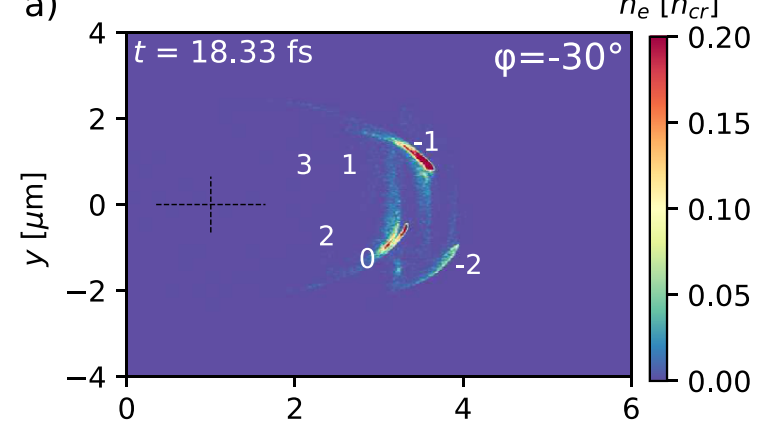

b)

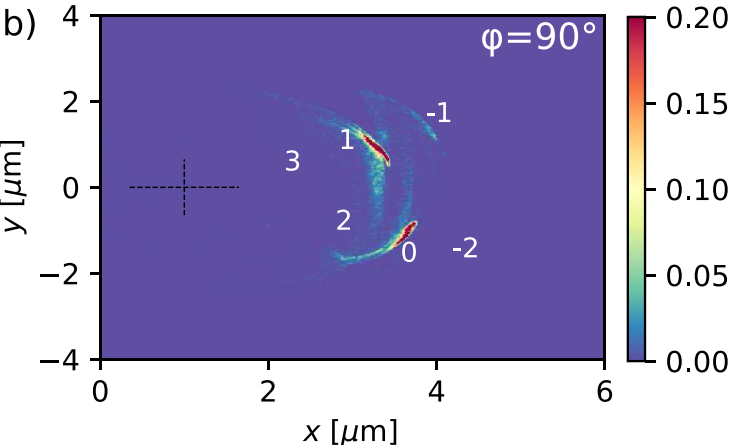

c )
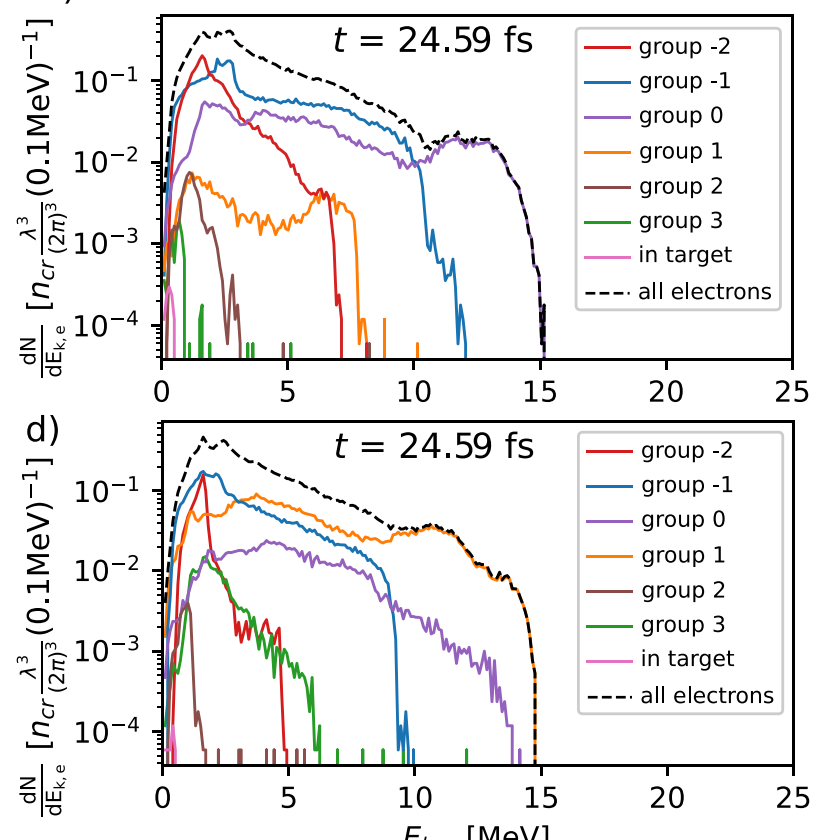

Figure 7. Densities ((a), (b)) and energy spectra ((c), (d)) of single electron bunches for initial values of phase $\varphi_{\mathrm{CEP}}=-30^{\circ}((\mathrm{a}),(\mathrm{c}))$ and $\varphi_{\mathrm{CEP}}=90^{\circ}((\mathrm{b}),(\mathrm{d}))$. Parameters as in figure 6 .

\subsection{Potential applications}

An isolated and dense attosecond electron bunch is a unique seed for a Thomson backscattering x-ray source of the similar duration. As the characteristic energy of the generated radiation is proportional to $E_{k, e}^{2}$ [4], the presented scheme is appropriate for that purpose even if the dominant bunch is not completely isolated, but has much higher energy. For example, in our original case (figures 5(a) and (d)), the energy of the neighbour bunches from the lower half-space is about a half of the dominant bunch cut-off energy. As a result, the related neighbour X-ray pulses would have a cut-off energy about four times lower than the main $\mathrm{x}$-ray pulse. Thus, an isolated attosecond $\mathrm{x}$-ray pulse could be easily energy filtered between the two photon energies in practical applications.

For an illustration, table 2 summarises the expected properties of the emitted $x$-rays calculated using the standard relations for linear $\left(a_{0, \mathrm{TS}}<1\right)$ and nonlinear $\left(a_{0, \mathrm{TS}} \gg 1\right)$ Thomson backscattering [4]. As a seed electron bunch, a part of the bunch No. 0 from figure 5(d) with $E_{k, e}>8 \mathrm{MeV}$ is considered. The intensity of the scattering pulse is varied, its length is set to 50 periods in order to obtain large number of photons and maintain the attosecond pulse duration in the same time [50].

Furthermore, a single electron bunch generation could enable the introduction of the relativistic mirror [51, 52] or other methods $[4,53]$ to produce bright and short photon bursts.

The potential secondary sources promise imaging applications with increased resolution especially in the temporal domain as the length of the electron sheet and thus of the secondary $\mathrm{x}$-ray pulse is limited by the factor of $\lambda / 2$.

Another potential application is to inject this single attosecond electron bunch into a laser wakefield accelerator [6]
Table 2. Properties of x-ray pulses by Thomson backscattering (TS) on electrons with $E_{k, e}>8 \mathrm{MeV}$ from spectrum in figure 5(d). There is $3.5 \times 10^{7}$ of such electrons. Fifty periods long TS pulse is considered. $a_{0, \mathrm{TS}}-\mathrm{TS}$ pulse strength, $E_{k, e}$-electron energy range, $E_{\mathrm{ph}}$-range of emitted photon energies, for $a_{0, \mathrm{TS}}>1$ it corresponds to critical energy range, $N$-total number of these photons.

\begin{tabular}{lclc}
\hline$a_{0, \mathrm{TS}}$ & $E_{k, e}$ range & $E_{\text {ph }}$ range & $N$ \\
\hline 0.1 & $8.0-15.5 \mathrm{MeV}$ & $1.6-6.8 \mathrm{keV}$ & $2.68 \times 10^{5}$ \\
0.5 & $8.0-15.5 \mathrm{MeV}$ & $1.6-6.8 \mathrm{keV}$ & $6.69 \times 10^{6}$ \\
2 & $8.0-15.5 \mathrm{MeV}$ & $2.4-10.1 \mathrm{keV}$ & $1.07 \times 10^{8}$ \\
4 & $8.0-15.5 \mathrm{MeV}$ & $4.8-20.2 \mathrm{keV}$ & $4.28 \times 10^{8}$ \\
\hline
\end{tabular}

near to the nanotarget. Such an electron injection scheme might result in a very short, relatively monoenergetic, highly relativistic electron bunch. The initial absolute energy spread of approximately $10 \mathrm{MeV}$ would not increase significantly as the short bunch duration secures the homogeneous acceleration of all electrons at the same phase of the wakefield. Also, the energy spread growth induced by the beam-loading effect [54] would be controlled under such circumstances with the bunch charge set by the size of the nanosphere. As a consequence, the final relative energy spread could be very low despite the initially broad energy spectrum.

\section{Conclusion}

The interaction of a few-cycle relativistic-intensity laser pulse with a mass-limited nanotarget enables the generation of a single attosecond relativistic electron bunch with continuous spectrum. The acceleration mechanism has two steps. First, electron bunches are ejected from the sub-micron overcritical 
nanodroplet with already relativistic energy due to the nanophotonic effect. Second, these bunches co-propagate with the laser pulse and are accelerated, as our results indicate, via the action of the transverse electric field and turned forward by the transverse magnetic field. The maximum acceleration energy scales with the square root of peak laser intensity in the investigated parameter regime.

A single bunch is generated even with pulses slightly longer than one optical cycle if the target parameters are adjusted to the laser intensity in a way that all the electrons are removed from the target at the latest by the laser period corresponding to peak intensity of the pulse. Under these conditions, our particle-in-cell simulations show electron bunches with continuous energy spectra reaching up to $2.4 a_{0}(\mathrm{MeV})$, carrying the charge of a few $\mathrm{pC}$ and their length being in the attosecond regime, down to 200 as. The required laser and target parameters are feasible with contemporary laser systems and commercially available mass-limited nanoparticles. Thus, it represents an excellent approach for generation of isolated attosecond $\mathrm{x}$ ray pulse via Thomson backscattering.

\section{Data availability statement}

The data that support the findings of this study are available upon reasonable request from the authors.

\section{Acknowledgments}

The authors are grateful to T Fülöp for fruitful discussions. The authors thank the two anonymous reviewers whose comments helped to improve and clarify this manuscript. This work received funding from the European Research Council (ERC) under the European Union's Horizon 2020 research and innovation programme under Grant Nos. 647121 and 787539. $\mathrm{L} \mathrm{V}$ acknowledges the support by the Swedish Research Council (2016-05409, 2019-02376 and 2020-05111).

Access to computing and storage facilities at Chalmers Centre for Computational Science and Engineering (C3SE) provided by the Swedish National Infrastructure for Computing is greatly appreciated. Simulations demanded during the peer-review process were performed on the Irene-Joliot-Curie machine hosted at TGCC, France, using High Performance Computing resources from GENCI-TGCC (Grant No. 2020x2016057678).

\section{ORCID iDs}

Vojtěch Horný (D) https://orcid.org/0000-0002-4510-3770

László Veisz (D) https://orcid.org/0000-0002-7694-9066

\section{References}

[1] Malka V, Faure J, Glinec Y, Rechatin C, Fuchs T, Oelfke U and Szymanowski H 2008 Commercial and Biomedical Applications of Ultrafast Lasers VIII vol 6881 (Int. Society for Optics and Photonics) p 68810B
[2] Gruse J-N et al 2020 Nucl. Instrum. Methods Phys. Res. A 983164369

[3] Gizzi L, Labate L, Baffigi F, Brandi F, Bussolino G, Fulgentini L, Koester P, Palla D and Rossi F 2015 Nucl. Instrum. Methods Phys. Res. B 355241

[4] Corde S, Ta Phuoc K, Lambert G, Fitour R, Malka V, Rousse A, Beck A and Lefebvre E 2013 Rev. Mod. Phys. 851

[5] Albert F and Thomas A G R 2016 Plasma Phys. Control. Fusion $\mathbf{5 8} 103001$

[6] Esarey E, Schroeder C and Leemans W 2009 Rev. Mod. Phys. 811229

[7] Gahn C, Tsakiris G, Pukhov A, Meyer-ter Vehn J, Pretzler G, Thirolf P, Habs D and Witte K 1999 Phys. Rev. Lett. 834772

[8] Gonsalves A et al 2019 Phys. Rev. Lett. 122084801

[9] Buck A, Nicolai M, Schmid K, Sears C M S, Sävert A, Mikhailova J M, Krausz F, Kaluza M C and Veisz L 2011 Nat. Phys. 7543

[10] Lundh O et al 2011 Nat. Phys. 7219

[11] Lifschitz A and Malka V 2012 New J. Phys. 14053045

[12] Beaurepaire B, Lifschitz A and Faure J 2014 New J. Phys. 16023023

[13] Tooley M, Ersfeld B, Yoffe S, Noble A, Brunetti E, Sheng Z, Islam M and Jaroszynski D 2017 Phys. Rev. Lett. 119044801

[14] Horný V, Petržílka V and Krůs M 2019 Plasma Phys. Control. Fusion 61085018

[15] Zhao Q, Weng S, Chen M, Zeng M, Hidding B, Jaroszynski D, Assmann R and Sheng Z 2019 Plasma Phys. Control. Fusion 61085015

[16] Zhu J, Assmann R, Dohlus M, Dorda U and Marchetti B 2016 Phys. Rev. Accel. Beams 19054401

[17] Kornaszewski A et al 2019 Relativistic Plasma Waves and Particle Beams as Coherent and Incoherent Radiation Sources III vol 11036 (Int. Society for Optics and Photonics) p 110360R

[18] Papp D, Hafz N and Kamperidis C 2019 Laser Part. Beams 37165

[19] Ferri J, Horný V and Fülöp T 2021 Plasma Phys. Control. Fusion 63045019

[20] Willingale L et al 2013 New J. Phys. 15025023

[21] Ma Y et al 2018 Proc. Natl Acad. Sci. 1156980

[22] Hussein A E et al 2021 New J. Phys. 23023031

[23] Jirka M, Vranic M, Grismayer T and Silva L 2020 New J. Phys. 22083058

[24] Shaw J et al 2021 Sci. Rep. 117498

[25] Andreev A and Platonov K Y 2013 Opt. Spectrosc. 114788

[26] Hu L-X, Yu T-P, Sheng Z-M, Vieira J, Zou D-B, Yin Y, McKenna P and Shao F-Q 2018 Sci. Rep. 87282

[27] Naumova N, Sokolov I, Nees J, Maksimchuk A, Yanovsky V and Mourou G 2004 Phys. Rev. Lett. 93195003

[28] Salamin Y I and Keitel C H 2002 Phys. Rev. Lett. 88095005

[29] Wang P, Ho Y, Yuan X, Kong Q, Cao N, Sessler A, Esarey E and Nishida Y 2001 Appl. Phys. Lett. 782253

[30] Pang J, Ho Y, Yuan X, Cao N, Kong Q, Wang P, Shao L, Esarey E and Sessler A 2002 Phys. Rev. E 66066501

[31] Liseykina T, Pirner S and Bauer D 2010 Phys. Rev. Lett. 104095002

[32] Popov K, Bychenkov V Y, Rozmus W, Sydora R and Bulanov S 2009 Phys. Plasmas 16053106

[33] Yu T-P, Hu L-X, Yin Y, Shao F-Q, Zhuo H-B, Ma Y-Y, Yang X-H, Luo W and Pukhov A 2014 Appl. Phys. Lett. 105114101

[34] Derouillat J et al 2018 Comput. Phys. Commun. 222351

[35] Thiele I, Skupin S and Nuter R 2016 J. Comput. Phys. 3211110

[36] Cardenas D, Ostermayr T, Di Lucchio L, Hofmann L, Kling M F, Gibbon P, Schreiber J and Veisz L 2019 Sci. Rep. 97321 
[37] Born M and Wolf E 2013 Principles of Optics: Electromagnetic Theory of Propagation, Interference and Diffraction of Light (Amsterdam: Elsevier)

[38] Di Lucchio L and Gibbon P 2015 Phys. Rev. Spec. Top.-Accel. Beams 18023402

[39] Pukhov A and Meyer-ter Vehn J 2002 Appl. Phys. B 74355

[40] Lin J, Batson T, Nees J, Thomas A G and Krushelnick K 2020 Sci. Rep. 1018354

[41] Wen M, Salamin Y I and Keitel C H 2020 Phys. Rev. Appl. 13034001

[42] Rivas D et al 2017 Sci. Rep. 75224

[43] Smijesh N, Zhang X, Fischer P, Muschet A, Salh R, Tajalli A, Morgner U and Veisz L 2019 Opt. Lett. 444028

[44] Kessel A et al 2018 Optica 5434
[45] Ouille M et al 2020 Light Sci. Appl. 947

[46] Toth S et al 2020 J. Phys. Photon. 2045003

[47] Microparticles GmbH (Berlin) (available at: www. microparticles-shop.de) (Accessed 15 October 2021)

[48] Ostermayr T et al 2018 Rev. Sci. Instrum. 89013302

[49] Hilz P et al 2018 Nat. Commun. 9423

[50] Khrennikov K, Wenz J, Buck A, Xu J, Heigoldt M, Veisz L and Karsch S 2015 Phys. Rev. Lett. 114195003

[51] Kiefer D et al 2013 Nat. Commun. 41763

[52] Hu L-X, Yu T-P, Li H-Z, Yin Y, McKenna P and Shao F-Q 2018 Opt. Lett. 432615

[53] Ma W et al 2014 Phys. Rev. Lett. 113235002

[54] Katsouleas T, Wilks S, Chen P, Dawson J M and Su J J 1987 Part. Accel. 2281 